\title{
Alpha Clustering and the stellar nucleosynthesis of carbon
}

\author{
H. Oberhummer, H. Krauss and K. Grün, T. Rauscher \\ Institut für Kernphysik, Technische Universität Wien, A-1040 Vienna, Austria \\ H. Abele, P. Mohr and G. Staudt \\ Physikalisches Institut, Univ. Tübingen, D-72076 Tübingen, Germany
}

\begin{abstract}
The astrophysical S-factor and reaction rates for the triple-alpha process are calculated in the direct-capture model. It is shown that the stellar carbon production is extremely sensitive to small variations in the $\mathrm{N}-\mathrm{N}$ interaction.
\end{abstract}

PACS: 24.50.+g, 25.20.Lj, 97.10.Cv

\section{Introduction}

The most important cluster reaction in nuclear astrophysics is certainly the triplealpha process occuring in helium burning of stars. Almost all of the carbon found in our universe is synthesized by this reaction. According to most biochemists belief, carbon is the only element that can form the basis of spontaneously generated life. Therefore, the stellar production of carbon is essential for the existence of life in our universe.

In the triple-alpha reaction three alpha particles merge in a two-step process to form the nucleus ${ }^{12} \mathrm{C}$. In the first step the ground state of ${ }^{8} \mathrm{Be}$ is formed. Even so the life time of this nucleus is extremly short $\left(\approx 10^{-16} \mathrm{~s}\right)$, it is anomalously long compared with the $\alpha+\alpha$ collision time $\left(\approx 10^{-21} \mathrm{~s}\right)$. Therefore, the ${ }^{8} \mathrm{Be}$ is almost in equilibrium with the $\alpha$-particles. During the life time of ${ }^{8} \mathrm{Be}$ a third $\alpha$-particle is captured by the reaction ${ }^{8} \mathrm{Be}(\alpha, \gamma){ }^{12} \mathrm{C}$. The first step depends sensitively on the properties of the ${ }^{8} \mathrm{Be}$ ground-state, whereas the reaction rate of the second step is mainly a consequence of the properties of the $0_{2}^{+}$state in ${ }^{12} \mathrm{C}$, which lies just above the threshold of the ${ }^{8} \mathrm{Be}(\alpha, \gamma){ }^{12} \mathrm{C}$ reaction.

\section{The triple-alpha process in direct capture}

A detailed account of the calculation and results for the astrophysical S-factor and the reaction rate of the triple-alpha process obtained in the direct-capture (DC) model is given in [1]. The $\alpha-\alpha$ scattering and the ground-state properties of ${ }^{8} \mathrm{Be}$ as well as the bound and resonant states in ${ }^{12} \mathrm{C}$, which are relevant for the triple-alpha process are discussed in [2].

Direct capture is based on the description of the dynamics of nuclear processes by a Schrödinger equation with local potentials in the exit channel. The most 
important ingredients of DC are the wave functions for the scattering states in the entrance channel and the and bound and quasi-bound (resonant) states in the entrance and exit channels. The renormalized folding potentials used in the calculation of the triple-alpha process can be found in [2].

The astrophysical S-factor for the reaction ${ }^{8} \mathrm{Be}(\alpha, \gamma){ }^{12} \mathrm{C}$ is calculated using the DC model [3], [4], [5] with the computer code TEDCA [6]. One observes a resonant contribution of the dominating $0_{2}^{+}$state at $0.2875 \mathrm{MeV}$ and a nonresonant background. These two contributions and the interference term are obtained simultaneously in our model. Approximating the cross section in the vicinity of the resonance energy of $E_{\mathrm{R}}=0.2875 \mathrm{MeV}$ by a Breit-Wigner formula we obtain the following widths: (i) $\Gamma\left(0_{2}^{+}\right) \approx \Gamma_{\alpha}\left(0_{2}^{+}\right)=7.5 \mathrm{eV}$ in agreement with the experimental data $\Gamma\left(0_{2}^{+}\right)^{\exp }=(8.3 \pm 1.0) \mathrm{eV}$ and (ii) $\Gamma_{\gamma}\left(0_{2}^{+}\right)=4.1 \mathrm{meV}$ which is comparable with the experimental value $\Gamma_{\gamma}\left(0_{2}^{+}\right)^{\exp }=(3.7 \pm 0.5) \mathrm{meV}$ [ [7].

We can now determine the reaction rates for the triple-alpha process at $10^{8} \mathrm{~K}$ using the results for the astrophysical S-factor calculated using the DC model of the foregoing sections. The low-temperature rates $\left(10^{7} \mathrm{~K}-10^{8} \mathrm{~K}\right)$ including the non-resonant parts can be found in 1].

\section{Variation of the nucleon-nucleon $(\mathrm{N}-\mathrm{N})$ interaction}

The following advantages in applying the DC model together with the folding procedure for the calculation of the astrophysical S-factor and the reaction rates for the triple-alpha process are evident. The first and second step of the process is carried out consistently using the same model, no parameter has to be adjusted to the reaction data and the resonant and non-resonant part of the astrophysical $\mathrm{S}$-factor is obtained simultaneously. These features also allow us to demonstrate the extreme sensitivity of the triple-alpha reaction to variations of the underlying effective $\mathrm{N}-\mathrm{N}$ interaction. A preliminary account of this calculation was given in [9].

We now introduce small variations of the strength of the effective $\mathrm{N}-\mathrm{N}$ interaction in the calculation of the ${ }^{8} \mathrm{Be}$-ground state and the $0_{2}^{+}$-state in ${ }^{12} \mathrm{C}$ without changing the renormalization factors $\lambda$ in the folding potentials given in [2]. These factors, introduced originally to describe higher-order corrections to the simple folding procedure, are insensitive to small variations of the effective $\mathrm{N}-\mathrm{N}$ interaction for states with a marked cluster structure. This result can be justified in the single-nucleon exchange approximation [10], [11]. In our calculations we also assume that the small variations of the effective $\mathrm{N}-\mathrm{N}$ interaction will only modify the $\alpha-$ density distribution needed in the computation of the folding potentials in higher order. Furthermore, the changes in the wave function of the final bound states in

${ }^{12} \mathrm{C}$ should also be negligible. The reason for this assumption is that changes of energies and wave functions are of the order of $|\Delta E / E| \lesssim\left|\left(\epsilon V_{0}\right) / E\right|$, where $\Delta E$ is 
Table 1: Effects of variations in the strength of the effective nucleon-nucleon interaction on the triple-alpha reaction rate.

\begin{tabular}{ccccccc}
\hline & $\begin{array}{c}E^{\mathrm{R}}[\mathrm{keV}] \\
{ }^{8} \text { Be g.s. }\end{array}$ & $\begin{array}{c}E^{\mathrm{R}}[\mathrm{keV}] \\
0_{2}^{+}\left({ }^{12} \mathrm{C}\right)\end{array}$ & $\begin{array}{c}\Gamma[\mathrm{eV}] \\
0_{2}^{+}\left({ }^{12} \mathrm{C}\right)\end{array}$ & $\begin{array}{c}F_{1} \\
1 . \text { step }\end{array}$ & $\begin{array}{c}F_{2} \\
2 . \text { step }\end{array}$ & $\begin{array}{c}F_{\mathrm{T}} \\
\text { total }\end{array}$ \\
\hline $0.2 \%$ & 66.8 & 215.5 & 0.2 & 18 & $3.7 \cdot 10^{3}$ & $6.7 \cdot 10^{4}$ \\
$0.1 \%$ & 79.2 & 251.7 & 1.5 & 4.2 & 60 & 250 \\
$0 \%$ & 91.5 & 287.5 & 7.5 & 1 & 1 & 1 \\
$-0.1 \%$ & 103.7 & 323.0 & 29 & 0.24 & 0.016 & 0.0038 \\
$-0.2 \%$ & 115.7 & 357.9 & 90 & 0.06 & $2.5 \cdot 10^{-4}$ & $1.5 \cdot 10^{-5}$ \\
\hline
\end{tabular}

the change in the energy, $E$ is the energy with respect to the threshold, $\epsilon$ is the change of the effective $\mathrm{N}-\mathrm{N}$ interaction and $V_{0}$ is the depth of the corresponding folding potential. Obviously, the above change is much smaller for states which have a large energy $|E|$ with respect to the threshold.

The changes of the resonance energy of the ground state of ${ }^{8} \mathrm{Be}$ as well as the resonance energy and total width $\Gamma$ of the $0_{2}^{+}$-state in ${ }^{12} \mathrm{C}$ induced by variations of the effective $\mathrm{N}-\mathrm{N}$ interaction are listed in Table 1 . It results that the $\gamma$-width of the $0_{2}^{+}$-state is almost unaffected by this variation. For the calculation of the reaction rates we will consider in the following only the resonant contributions. For a temperature of $1 \cdot 10^{8} \mathrm{~K}$ the changes of the reaction rate of the first, second and both steps in the triple-alpha process are given by $F_{1}$ and $F_{2}$ together with $F_{\mathrm{T}}=F_{1} \cdot F_{2}$, respectively and are listed in the last three columns of Table 1 .

As can be seen from this table the reaction rates of the triple-alpha process are extremely sensitive to small variations in the strength of the effective $\mathrm{N}-\mathrm{N}$ interaction. A variation of this interaction and therefore also of the depth of the folding potentials $\left(V_{0} \approx 100 \mathrm{MeV}\right)$ by about $0.1 \%$ leads to a shift of the ${ }^{8} \mathrm{Be}$ ground-state and the $0_{2}^{+}$-state in ${ }^{12} \mathrm{C}$ of the order of $10-30 \mathrm{keV}$. These energie shifts introduced in the Boltzmann-factors $\exp \left(-E_{\mathrm{R}} / k T\right)$ for the first and second step of the triplealpha proces result then in the drastic changes of the reaction rates shown in Table 1.

\section{The anthropic principle and the triple-alpha process}

The "anthropic principle" [12] states that of all possible universes the one we actually inhabit is tailor-made for the creation of life. This subject has been extensively reviewed in [13]. The triple-alpha process plays a key role for the anthropic principle. This can be seen from the remarkable prediction made by F. Hoyle that the creation of carbon would only be possible, if this reaction would proceed resonantly. This to our knowledge is the only case, where the anthropic principle was used to predict the outcome of a laboratory experiment. Later on, the prediction of the 
$0_{2}^{+}$-state in ${ }^{12} \mathrm{C}$ was indeed then confirmed by experiment [14], [15], [16].

Investigations of the changes in nucleosynthesis of very light elements by variations of the coupling constants have already been performed for the unbinding of the deuteron and binding of the di-proton 17]. In this case the limit was about $16 \%$ for the deuteron and about $12 \%$ for the di-proton in order that the anthropic principle is of significance of such variations. In the case of the triple-alpha process we showed that a variation of the coupling constants by only $0.1 \%$ would already be of significance for the anthropic principle, because it reduces the carbon production by about a factor of 250 at $10^{8} \mathrm{~K}$.

Acknowledgments: We want to thank the Austrian Science Foundation (FWF)

(project P8806-PHY), the Österreichische Nationalbank (project 3924) and the DFG-project Sta290/2.

\section{References}

[1] Krauss, H., Grün, K., Herndl, H., Oberhummer, H., Abele, G., Mohr, P., Staudt, G.: submitted to Ap. J.

[2] Mohr, P., Abele, H., Kölle V., Staudt, G., Oberhummer, H., Krauss, H.: this volume

[3] Oberhummer, H., Staudt G.: in Nuclei in the Cosmos. Ed. H. Oberhummer, p. 29. Berlin: Springer-Verlag 1991

[4] Mohr, P., Abele, R. Zwiebel, G., Staudt, G., Krauss, H., Oberhummer, H. Denker A., Hammer J. W., Wolf, G.: submitted to Phys. Rev. C

[5] Kim, K. H., Park, M. H., Kim, B. T.: Phys. Rev. C23, 363 (1987)

[6] Krauss, H.: computer code TEDCA, TU Wien, unpublished

[7] Ajzenberg-Selove, F.: Nucl. Phys. A506, 1 (1990)

[8] Rolfs, C., Rodney, W. S.: Cauldrons in the Cosmos. Chicago: University of Chicago Press 1988

[9] Oberhummer, H., Grün, K., Hinterberger, R., Krauss, H., Abele, H., Staudt, G., Belyaev, V.: in Proceedings of the Seventh Workshop in Nuclear Astrophysics 22.-27. March 1993, Ringberg Castle, Bad Wiessee. Eds. E. Mller, W. Hillebrandt. Garching: Max-Planck-Institut fr Physik und Astrophysik, in press

[10] Satchler, G. R., Love, W. G.: Phys. Rep. 55, 183 (1979)

[11] Love, W. G., Owen, L. W.: Nucl. Phys. A239, 74 (1975) 
[12] Carter, B.: in Confrontation of Cosmological Theories with Observation, ed. M. S. Longmaier, Dordrecht: Reidel 1974

[13] Barrow, J. D., Tipler, F. J.: The Anthropic Cosmological Principle. Oxford: Clarendon Press 1986

[14] Dunbar, D. N. F., Pixley, R. E., Wenzel, W. A., Whaling, W.: Phys. Rev. 92, $649(1953)$

[15] Hoyle, F., Dunbar, D. N. F., Wenzel, W. A., Whaling, W.: Phys. Rev. 92, 1095 (1953)

[16] Hoyle, F.: Astroph. J. Suppl. 1, 121 (1954)

[17] Pochet, T., Pearson, J. M., Beaudet, G., Reeves H.: Astron. and Astrophys. 243, 1 (1991)

[18] Livio, M., Hollowell, D., Weiss, A. Truran, J. W.: Nature 340, 281 (1989) 